\title{
Determinants of the Epithelial-Muscular Axis on Embryonic Stem Cell-Derived Gut-Like Structures
}

\author{
Yi Luo $^{a}$ Miyako Takakib Hiromi Misawab Hiroko Matsuyoshib \\ Tomonori Sasahira $^{a}$ Yoshitomo Chihara ${ }^{a}$ Kiyomu Fujii ${ }^{a}$ Hitoshi Ohmori $^{a}$ \\ Hiroki Kuniyasu $^{a}$ \\ Departments of a Molecular Pathology and ${ }^{b}$ Physiology II, Nara Medical University, Kashihara, Japan
}

\section{Key Words}

Embryonic stem cells · Hedgehog • Epithelial-muscular axis phosphatase in the embryoid bodies. These findings suggest that the motility of the ES gut depends on the domes' epithelial-muscular axis.

Copyright $\odot 2010$ S. Karger AG, Basel

\begin{abstract}
Dome-like structures with epithelial-muscular layers resembling the gut have been derived from mouse embryonic stem (ES) cells. These domes have been reported to show spontaneous contractions and are called ES gut. In the present study, we examined the epithelial-muscular axis of these domes by detecting differentiation markers. A normal epithelial-muscular axis was exhibited in the domes with spontaneous motility, whereas the domes without spontaneous motility showed either an inverted or obscure axis. To investigate the factors affecting the epithelial-muscular axis, we examined the expression of hedgehog signaling factors in the domes. Expression of hedgehog family factors was detected in the epithelial components of the domes with motility, whereas this expression was inverted or obscure in the domes without motility. Out of the 25 domes, 10 of the 10 motility (+) domes showed a normal epithelial-muscular axis, whereas 14 of the 15 motility (-) domes lacked a normal epithelial-muscular axis. This implies that activin A upregulated the expression of sonic hedgehog and intestinal alkaline
\end{abstract}

\section{Introduction}

Embryonic stem (ES) cells are pluripotent cells which retain the potential to differentiate diverse tissues and organs [1]. ES cells are capable of generating spontaneous constructions, including dome-like structures (domes), consisting of epithelial cells, smooth muscle cells, interstitial cells of Cajal (ICC), and a few immature neurons, which bear a histological resemblance to the gut $[2,3]$. This gut-like structure has been called 'gut' from mouse ES cells (i.e. ES gut). In this study, we grew ES gut using a hanging drop culture followed by an outgrowth culture $[2,3]$. After 4 days in hanging drop cultures, the ES cells formed small spheroid structures called embryoid bodies (EBs). After 7 days, the EBs were transferred to outgrowth cultures. Two weeks later, the EBs had spontaneously given rise to domes with hollow inner cavities.

\section{KARGER}

Fax +41613061234 E-Mail karger@karger.ch www.karger.com

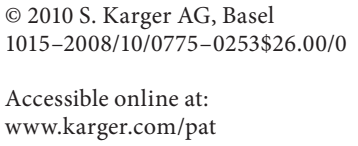


The gut is a highly integrated structure comprising many tissues. In gut embryogenesis, precursor cells form a primitive gut through processes involving mesendoderm formation, endoderm formation, and gastrulation $[4,5]$. Unlike normal gut embryogenesis, ES gut formation lacks 3-dimensional processes. Therefore, ES gut cell differentiation may be limited due to a lack of topological cell-to-cell interaction. Indeed, the yield rate of ES gut formation is not $100 \%$. Some domes exhibit highly coordinated periodic contractions and relaxations, whereas some fail to exhibit spontaneous motility.

To explore which structural components are required for the formation of functional ES gut, we examined the relationships between epithelium, muscle, nerve, and ICC and the rates of cell proliferation and cell apoptosis in the domes. Furthermore, we examined the mechanisms governing the domes' histological structure. Finally, we examined the expressions of hedgehog $(\mathrm{HH})$ homolog factors in $\mathrm{HH}$ signaling pathways. Sonic $\mathrm{HH}(\mathrm{SHH})$ function is essential for cell fate specification as well as for growth and patterning direction during embryonic development [6]. In this study, we examined the functional structure of the motility $(+)$ ES gut and elucidated the factors affecting epithelial-muscular axis formation.

\section{Materials and Methods}

\section{Culture of ES Cells and Formation of ES Gut}

Undifferentiated ES cells (EB3) were maintained on gelatincoated dishes without feeder cells in Dulbecco's modified Eagle's medium (DMEM; Sigma Chemical Co., St. Louis, Mo., USA) supplemented with $10 \%$ fetal bovine serum (FBS; GIBCO/BRL, Grand Island, N.Y., USA), 0.1 mM 2-mercaptoethanol (Wako, Tokyo, Japan), $0.1 \mathrm{~mm}$ nonessential amino acids (GIBCO/BRL), $1 \mathrm{mM}$ sodium pyruvate (Bio-Whittaker), and 1,000 U/ml LIF (Chemicon, Temecula, Calif., USA) [2]. The EB3 cells (a kind gift from Dr. Hitoshi Niwa of the Riken Center for Developmental Biology, Kobe, Japan) carried the blasticidin S-resistant selection marker gene driven by the Oct-3/4 promoter (active in undifferentiated cells) and were maintained in medium containing $10 \mu \mathrm{g} /$ $\mathrm{ml}$ blasticidin S to eliminate differentiated cells [7]. To induce EB formation, dissociated ES cells were cultured in hanging drops as previously described, with minor modifications. Two weeks later, they were shown to spontaneously give rise to a dome, i.e. the ES gut. To induce EB formation, we cultured dissociated ES cells in hanging drops $[1,8]$, with minor modifications. The cell density of 1 drop was 500 cells per $15 \mu$ l of ES cell medium in the absence of LIF. After 6 days in a hanging drop culture, the resulting EBs were plated onto plastic $100-\mathrm{mm}$ gelatin-coated dishes and allowed to attach for the outgrowth culture $[7,9,10]$. Culturing in hanging drops was the most important process for differentiating ES gut. Each ES cell-cluster underwent a dramatic transformation into a dome with a solid and cavity-like structure, and some of them showed ambiguous beating. On approximately day 14 , these clusters proliferated to form more prominent 3-dimensional constructions with a cystic structure and began rhythmic contractions that were not necessarily frequent or regular. On approximately day 21, the clusters (ES gut) showed coordinated contraction patterns with relatively regular rhythms, although there were some incomplete domes even on day 21 .

Immunohistochemistry

For the immunohistochemistry of thin sections, ES gut was fixed with $4 \%$ paraformaldehyde at $4^{\circ} \mathrm{C}$ and then scraped from the culture dish and embedded into $0.6 \%$ agarose-PBS, which was subsequently embedded in a paraffin block in the usual manner. Consecutive $4-\mu \mathrm{m}$ sections were cut from each block, subjected to antigen retrieval with pepsin (DAKO Corp., Carpinteria, Calif., USA) for $20 \mathrm{~min}$ at room temperature, and immunostained using the immunoperoxidase technique. After the blockade of endogenous peroxidase activity by incubation for $15 \mathrm{~min}$ in a $3 \% \mathrm{H}_{2} \mathrm{O}_{2}$ methanol solution, the sections were rinsed with PBS and incubated with a primary antibody diluted with washing solution (BioGenex, San Ramon, Calif., USA) for $2 \mathrm{~h}$ at room temperature. Thereafter, they were rinsed again with PBS and incubated for $1 \mathrm{~h}$ at room temperature with a peroxidase-conjugated secondary antibody diluted to $0.5 \mu \mathrm{g} / \mathrm{ml}$ (Medical \& Biotechnological Laboratories Co., Ltd., Nagoya, Japan). All sections were then rinsed with PBS, color-developed using diaminobenzidine solution (DAKO), washed in water, and counterstained with Meyer's hematoxylin (Sigma). Care was taken to ensure that the antibody reaction and DAB exposure were the same for all specimens. The antibodies used in the primary reaction are listed in table 1.

\section{Enzyme-Linked Immunosorbent Assay}

For the enzyme-linked immunosorbent assay (ELISA), $1 \times$ $10^{5}$ cells were cultured using a hanging drop method in $0.5 \mathrm{ml}$ of stem cell medium (Sigma) with or without recombinant human/ mouse/rat acitivin A (100 ng/ml; Cedarlane Laboratories Ltd., Burlington, N.C., USA) for 24 h. The cultured medium was filtered through a $0.2-\mu \mathrm{m}$ filter (Becton-Dickinson, Bedford, Mass., USA). The filtered medium was used for ELISA. Concentrations of SHH and intestinal alkaline phosphatase (IAP) were detected using a Sonic Hedgehog DuoSet ELISA development kit (R\&D Systems, Inc.) and an alkaline phosphatase, intestine, ELISA kit (Uscn Life Science, Inc., Wuhan, China), respectively, according to the provider's instruction.

Statistical Analysis

All statistics were carried out with StatView version 4.5 (SAS Institute, Inc., Cary, N.C., USA), and $\mathrm{p}<0.05$ was considered statistically significant.

\section{Results}

\section{Dome Motility}

As shown in figure 1a, domes were found after the EBs had been in the outgrowth culture for 2 weeks. On fluorescent calcium ion probe imaging, motility $(+)$ domes showed spontaneous intracellular $\mathrm{Ca}^{2+}$ oscillations (fig. $1 b)$, whereas motility (-) domes did not. 
Fig. 1. Calcium oscillation in ES gut. a Fluorescent image of ES gut. Bar $=100 \mu \mathrm{m}$. Mouse ES cells formed a dome-like structure with a hollow inner cavity at 2 weeks after outgrowth culture. b The intracellular calcium concentration was detected by a fluorescent probe. Calcium oscillation was also detected. The upper panel displays a spatial view and the lower panel a temporal view.
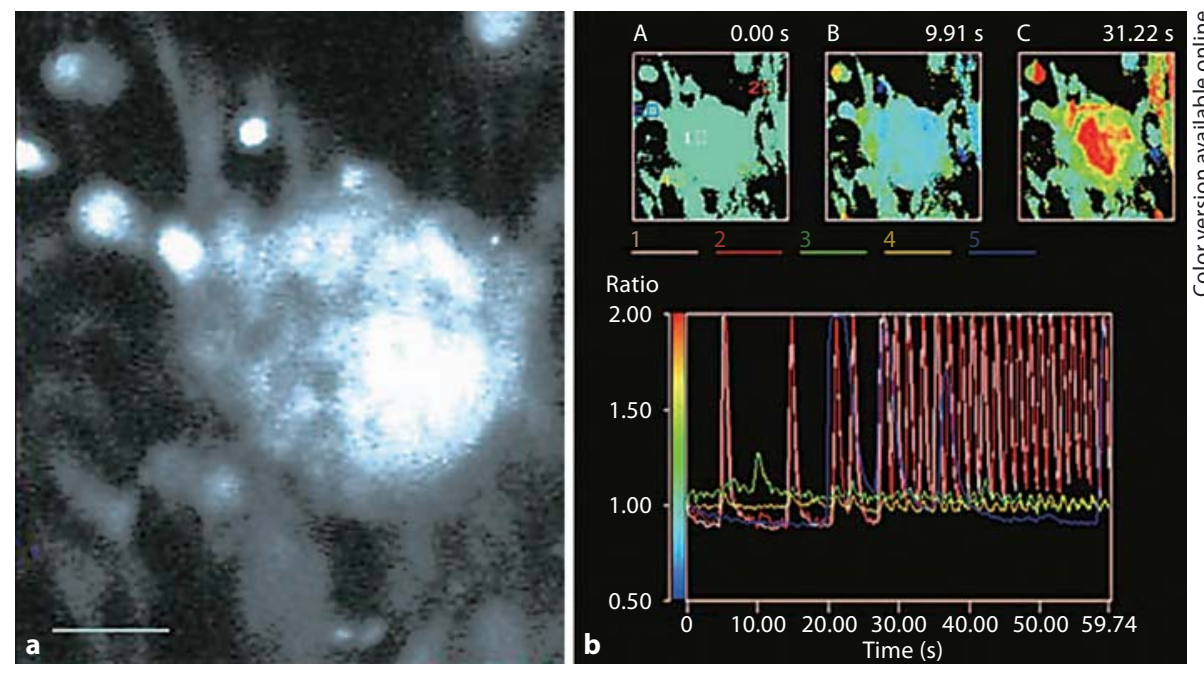

Table 1. Antibodies used for immunohistochemical analysis

\begin{tabular}{|c|c|c|c|c|}
\hline Antibody & Abbreviation & Company & Clone & Marker \\
\hline Cytokeratin & $\mathrm{CK}$ & Zymed $^{1}$ & & epithelium \\
\hline$\alpha$-Smooth muscle actin & SMA & Zymed & & smooth muscle \\
\hline c-Kit & & Santa Cruz ${ }^{2}$ & C-19 & ICC \\
\hline TFF3 & TFF3 & NanoTools ${ }^{3}$ & & intestine \\
\hline Ki67 & & DAKO & & proliferation \\
\hline ss-DNA & ssDNA & DAKO & & apoptosis \\
\hline Sonic hedgehog & $\mathrm{SHH}$ & Santa Cruz & H-160 & $\mathrm{HH}$ ligand \\
\hline Patched & PTC & Santa Cruz & G-19 & HH inhibitory receptor \\
\hline Smoothened & SMO & Santa Cruz & N-19 & HH excitatory receptor \\
\hline Glioma-associated Oncogene 1 & Gli1 & Rockland $^{4}$ & & $\mathrm{HH}$ nuclear messenger \\
\hline
\end{tabular}

${ }^{1}$ Zymed Laboratories, Inc., San Francisco, Calif., USA. ${ }^{2}$ Santa Cruz Biotechnology, Inc., Santa Cruz, Calif., USA. ${ }^{3}$ NanoTools, Teningen, Denmark. ${ }^{4}$ Rockland Immunochemicals, Inc., Gilbertsville, Pa., USA.

\section{Expression of Differentiation Markers in Domes}

We examined the distribution of tissue components cytokeratin (CK), $\alpha$-smooth muscle actin (SMA), c-Kit, trefoil factor 3 (TFF3), Ki67, and ssDNA in motility $(+)$ domes as compared to motility (-) domes (fig. 2; table 2). Motility (+) domes showed a CK-positive epithelial component on their inner surface and an SMA-positive muscle component in their outer layer, whereas motility (-) domes showed an obscure distribution of CK-positive cells on their outer surface and SMA-positive cells throughout the whole wall. Motility $(+)$ domes showed scattered c-Kit-positive ICC-suspected cells in their outer layers. However, motility (-) domes demonstrated a distribution of only a few c-Kit-positive cells. In terms of cell growth, motility (+) domes showed Ki67-positive prolif- erating cells and ssDNA-positive apoptotic cells mainly in their inner layer, which coincided with a CK-positive cell distribution. In contrast, motility (-) domes showed numerous Ki67-positive cells in both their inner and outer layers, and ssDNA-positive apoptotic cell debris was found within their cavities. TFF3 immunoreactivity, an intestinal epithelial marker, was found on the inner surfaces of motility $(+)$ domes, whereas motility (-) domes showed TFF3 immunoreactivity on their outer surfaces.

In 25 domes, CK, SMA, TFF3, Ki67, and ssDNA showed opposite distributions of immunoreactive cells between motility (+) and motility (-) domes (table 2 ). cKit-positive cells were more frequently detected in motility $(+)$ domes than in motility $(-)$ domes. 
Fig. 2. Expression of differentiation markers in motility (+) and motility (-) domelike structures. Differentiation of epithelium, smooth muscle, nerve, ICC, and intestinal mucosa, as well as cell proliferation, and apoptosis were assessed by the expressions of CK, SMA, c-Kit, TFF3, Ki67, and ssDNA, respectively. Bar $=50 \mu \mathrm{m}$.

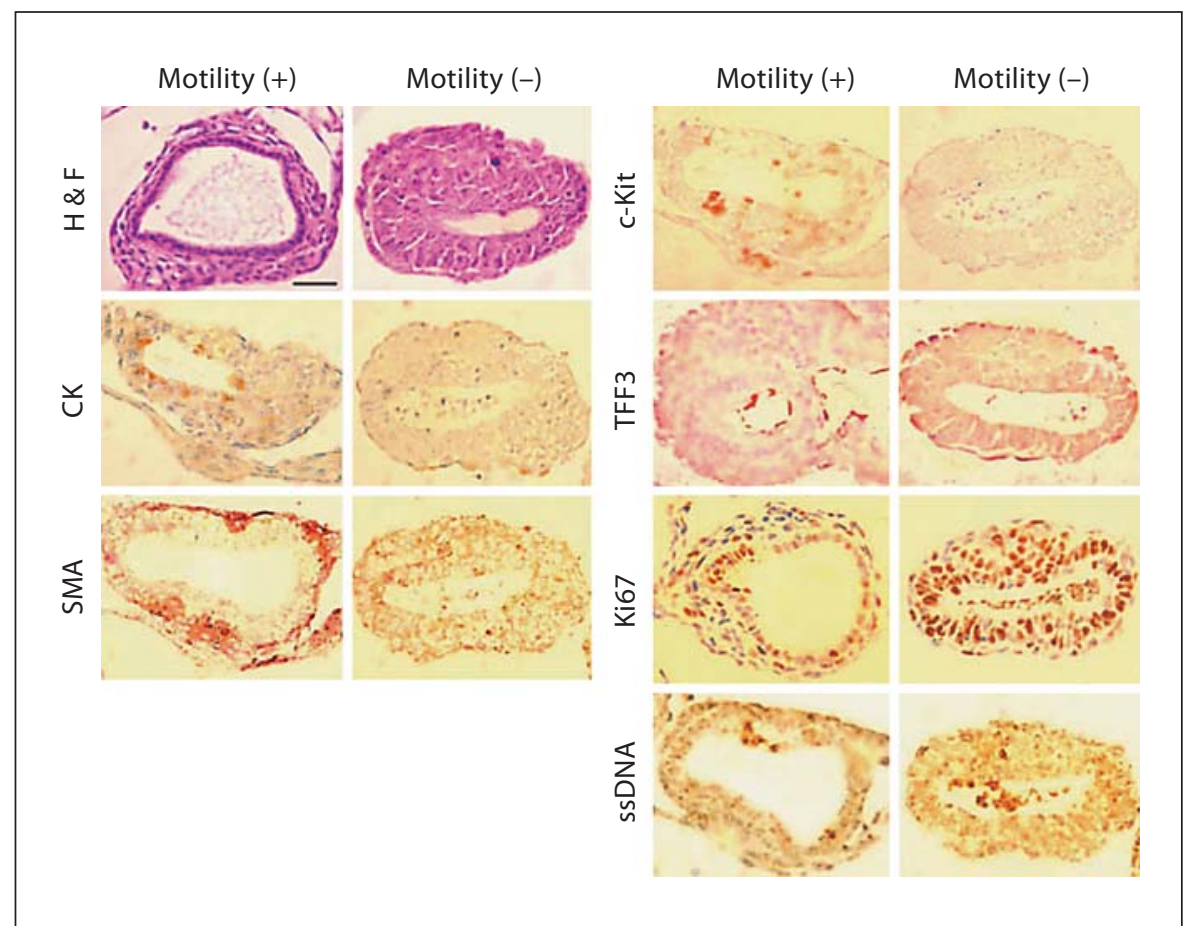

Table 2. Expression of differentiation markers in motility $(+)$ and motility (-) dome-like structures

\begin{tabular}{lllll}
\hline & Layer & $\begin{array}{l}\text { Motility (+) } \\
(\mathrm{n}=10)\end{array}$ & $\begin{array}{l}\text { Motility }(-) \\
(\mathrm{n}=15)\end{array}$ & p value \\
\hline $\mathrm{CK}^{1}$ & Inner & 10 & 1 & $<0.0001$ \\
& Outer & 0 & 14 & 0.0066 \\
$\mathrm{SMA}^{1}$ & Inner & 0 & 14 & \\
& Outer & 10 & 15 & 0.0102 \\
$\mathrm{C}^{-K i t}{ }^{2}$ & & $10(12 \pm 4$ cells $)$ & $3(4 \pm 3$ cells $)$ & 0.0001 \\
TFF3 $^{1}$ & Inner & 10 & 1 & $\mathrm{NS}$ \\
& Outer & 0 & 14 & $<0.0001$ \\
$\mathrm{Ki}^{3}{ }^{3}$ & Inner & $76 \pm 12 \%$ & $86 \pm 14 \%$ & $<0.0001$ \\
& Outer & $12 \pm 3 \%$ & $92 \pm 16 \%$ & $<0.0001$ \\
ssDNA $^{3}$ & Inner & $5 \pm 2 \%$ & $16 \pm 4 \%$ & \\
& Outer & $1 \pm 1 \%$ & $22 \pm 5 \%$ & \\
& & & &
\end{tabular}

${ }^{1}$ When the positivity of immunoreactive cells was $30 \%$ or more, it was judged as positive. ${ }^{2}$ Positive cell number in a dome. ${ }^{3}$ Labeling index. NS $=$ Not significant.

\section{Relationship between Motility Status and the \\ Epithelial-Muscular Axis}

To confirm the significance of the epithelial-muscular axis in the motility of ES gut, we examined 25 domes (table 3). In these 25 domes, a normal epithelial-muscular
Table 3. Relation of the histological axis and motor activity of ES gut

\begin{tabular}{lll}
\hline & \multicolumn{2}{l}{ Motility } \\
\cline { 2 - 3 }$(+)(\mathrm{n}=10)$ & $(-)(\mathrm{n}=15)$ \\
\hline $\begin{array}{l}\text { Epithelial-muscle axis } \\
\text { Positive }\end{array}$ & 10 & 1 \\
Negative & 0 & 14
\end{tabular}

Motility status vs. epithelial-muscular axis: $\mathrm{p}<0.0001$.

axis was found in all 10 motility (+) domes, whereas a normal axis was found in only 1 of the 15 motility (-) domes $(\mathrm{p}<0.0001)$. The single motility $(-)$ dome with a normal epithelial-muscular axis contained very few cells expressing c-Kit. These results suggest that the motility (+) domes exhibited an inner epithelial-outer muscular cell distribution (epithelial-muscular axis) with a differentiation of ICCs. In contrast, motility (-) domes exhibited an obscure inverted muscular-epithelial axis. Motility (-) domes also had high levels of proliferation and apoptosis which suggested an immature property. 


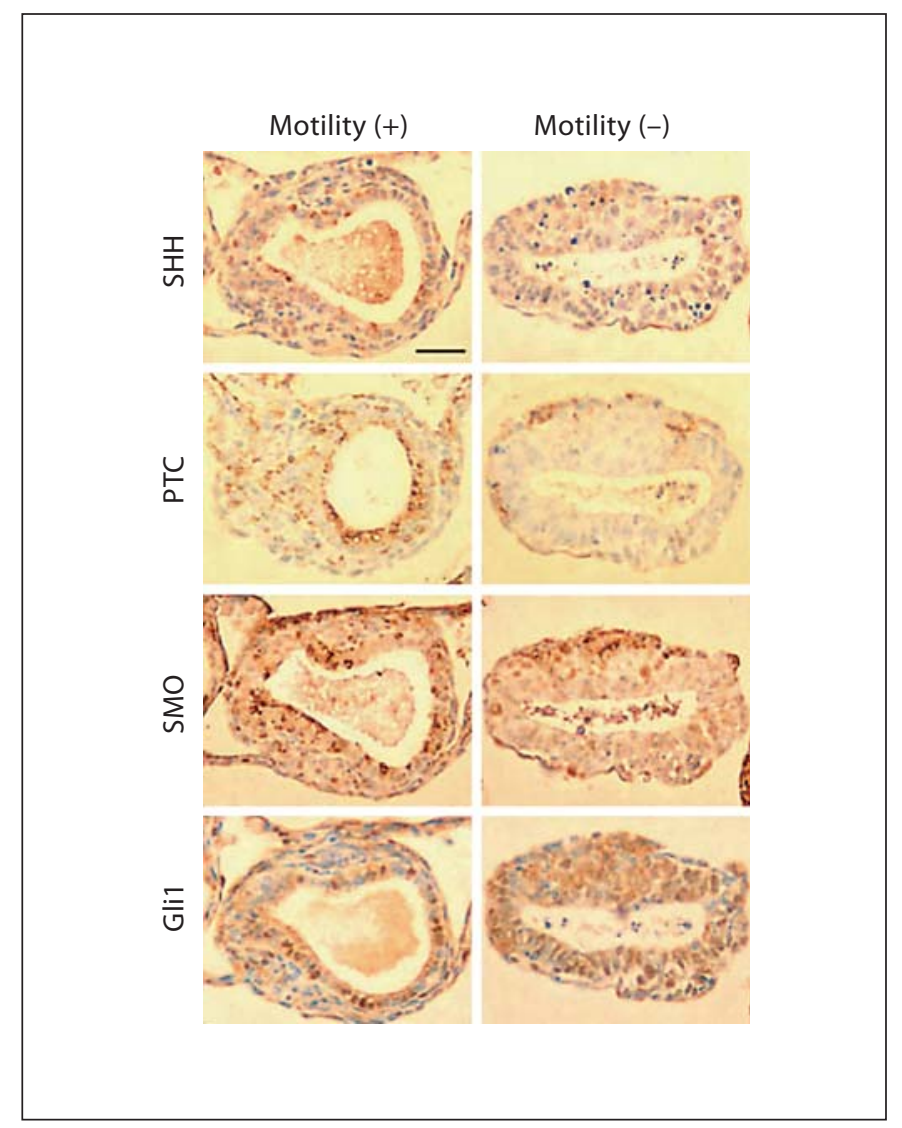

Fig. 3. Expression of $\mathrm{HH}$ factors and Wnt pathways in motility $(+)$ and motility (-) dome-like structures. Expression of $\mathrm{HH}$ pathway factors (SHH, PTC, SMO, and Gli1) was detected in motility (+) and motility (-) dome-like structures. Bar $=50 \mu \mathrm{m}$.

\section{Expression of HH Signaling Factors in the ES Gut}

In order to examine the underlying mechanism generating the epithelial-muscular axis, we examined the expression of $\mathrm{HH}$ signaling factors $\mathrm{SHH}, \mathrm{PTC}, \mathrm{SMO}$, and Gli1) in the domes (fig. 3; table 4). In motility (+) domes, $\mathrm{SHH}, \mathrm{PTC}, \mathrm{SMO}$, and Glil were expressed in the inner epithelial layer, whereas motility (-) domes showed $\mathrm{SHH}$, PTC, and SMO in the outer layer and Glil in the whole wall. The single case of a motility (-) dome without the axis showed a normal distribution of $\mathrm{SHH}$ but not of PTH, SMO, or Gli1.

\section{Effects of Activin A on the Activation of $\mathrm{SHH}$}

Activin A activates SHH in mouse embryos [11] and EBs [12]. In order to assess the effects of activin A on the domes' histological axis formation (fig. 4), we added activin A to the medium of the EB culture. Activin A increased the number of $\mathrm{SHH}$-expressing cells in day- $4 \mathrm{EBs}$
Table 4. Expression of hedgehog family factors in motility (+) and motility (-) dome-like structures

\begin{tabular}{llccc}
\hline & Layer & $\begin{array}{c}\text { Motility }(+) \\
(\mathrm{n}=10)\end{array}$ & $\begin{array}{l}\text { Motility }(-) \\
(\mathrm{n}=15)\end{array}$ & p value \\
\hline $\mathrm{SHH}^{1}$ & Inner & $10^{1}$ & 1 & $<0.0001$ \\
& Outer & 0 & 14 & \\
PTH & Inner & 10 & 0 & $<0.0001$ \\
& Outer & 0 & 15 & $<0.0001$ \\
SMO & Inner & 10 & 0 & 0.0274 \\
& Outer & 0 & 15 & \\
& Inner & 10 & 14 & \\
& Outer & 1 & 15 &
\end{tabular}

${ }^{1}$ When the positivity of immunoreactive cells was $30 \%$ or more, it was judged as positive.

as compared to untreated day-4 EBs (fig. 4a). Subsequently, the concentrations of $\mathrm{SHH}$ were examined in EBs (fig. 4b, c). Activin A treatment increased the SHH in EBs 11.8-fold. Finally, we examined the effect of activin A on intestinal differentiation. The concentration of IAP, which is an intestinal differentiation marker [13], was increased 4.6-fold in activin A-treated EBs.

\section{Discussion}

In this study, we examined the motility and structure of the ES gut. Our results demonstrate that epithelialmuscular axis formation is essential for the motility of domes. We examined the distribution of the tissue components in the motility (+) and motility (-) domes using immunohistochemistry, and our results showed that a normal epithelial-muscular axis was found in the motility (+) domes but not in the motility (-) domes. Motility $(-)$ domes showed an inverse axis and immature differentiation. Differentiation of ICC was found concurrently with normal epithelial-muscular axis formation in the dome walls. Furthermore, the $\mathrm{HH}$ signal, induced by activin A, played a pivotal role in epithelial-muscular axis formation. Recently, the gut-like structure was also generated from induced pluripotent stem (iPS) cells using the same methodology as with our ES gut [14]. Our findings might be applicable to iPS-derived gut-like structures.

The embryogenesis of the gut is a complicated process. The precursor cells of the gut are derived from epiblasts which form a primitive streak with mesendoder- 

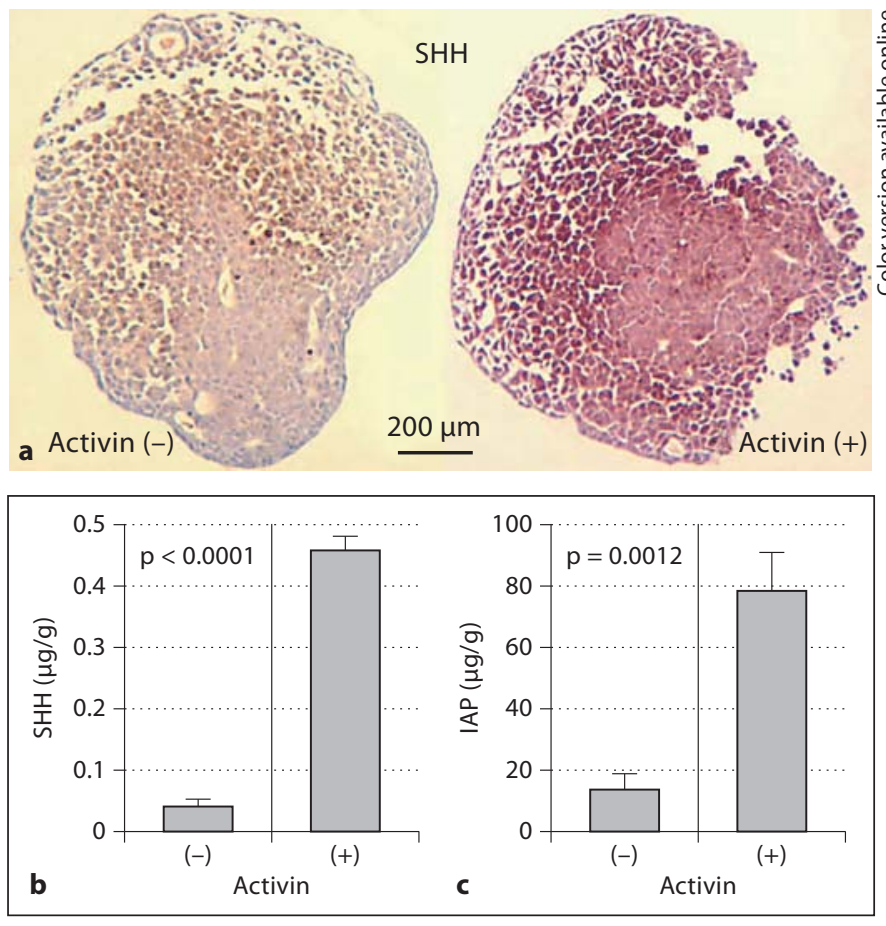

Fig. 4. Effects of activin A on SHH expression and intestinal differentiation. EBs were formed from ES cells in hanging drop cultures with or without activin A treatment (100 ng/ml). a Immunohistochemistry of SHH in day- 4 EBs. Bar $=200 \mu \mathrm{m}$. Concentrations of SHH (b) and IAP (c) in day- 4 EBs were detected by ELISA. Bars represent SD.

mal features [4]. The embryonic endoderm is generated at the ventral surface of the embryo through gastrulation from the primitive streak [5]. The embryonic endoderm forms a tube from the single cell layer sheet [5]. This multistep process of gut embryogenesis contains cell differentiation, cell-cell interaction, and spatiotemporal cognition. In contrast, ES gut genesis from ES cells retains cell differentiation and cell-cell interaction but fails to include spatiotemporal cognition. Our study showed that the concurrent expression of $\mathrm{HH}$ factors and Wnt pathways in the inner layer of the motility $(+)$ domes were associated with epithelial differentiation. $\mathrm{SHH}$ is a known inducer of intestinal differentiation in the endoderm and induces intestinal fatty acid-binding protein [11].

Both $\mathrm{SHH}$ and bone morphogenetic protein-4 are involved in the development of the cell-renewable epithelium [15]. SHH is an important endodermal signal in the endodermal-mesodermal cross-talk that patterns the developing gut tube along different axes [16]. Through epi- thelial-mesenchymal interactions, $\mathrm{HH}$ signaling ensures the appropriate axial patterning of the embryonic gut [6]. Thus, activation of $\mathrm{HH}$ pathways is associated with normal epithelial-muscular axis formation in the ES gut. Mutations in $\mathrm{SHH}$ and downstream signaling molecules are known to cause a variety of the congenital malformations of the gastrointestinal tract, including esophageal atresia, tracheoesophageal fistula, annular pancreas, midgut malrotation, and duodenal and anal atresia [16]. TGF- $\beta$ and FGF9 also play roles in intestinal development, which should be the subject of our further studies $[17,18]$.

Unlike tube formation in the embryonic endoderm, internal space formation in the ES gut is poorly understood. However, apoptotic cell debris was found in the internal spaces of the motility (-) domes, suggesting that these spaces are pseudolumens. Since the motility (-) domes showed higher proliferative activity than the motility $(+)$ domes, rapid cell cycling might induce a pseudolumen in domes without a mature functional histological structure.

Activin is a specific determinant of endodermal and mesodermal formation. Previously, by Xenopus animal cap assay, it was demonstrated that high concentrations of activin induce endomesoderm formation, whereas low concentrations of activin induce mesoderm formation [19]. Moreover, high concentrations of activin induce hepatocyte differentiation from the mesendoderm. In contrast, low concentrations of activin induce skeletal muscle differentiation [20]. Activin A can promote intestinal differentiation via an SHH-dependent mechanism [11]. Our data also showed an increase in $\mathrm{SHH}$ production and an acceleration of intestinal differentiation by activin A treatment. Activin also activates the c-Kit pathway [21] which is expressed in the ICC.

In mature gut, ICCs are essential for spontaneous intestinal motility [22]. In ES gut, spontaneous rhythmic contractions depend on the ICC network in the smooth muscle layer [3]. Moreover, inhibition of c-Kit by imatinib abrogates ICC formation and spontaneous motility in ES gut [23]. In the present study, ICCs were well developed in motility (+) domes. What is the factor affecting ICCs development? ICCs are derived from a common mesenchymal origin with smooth muscle cells [24]. Maturation of both ICCs and gut smooth muscle cells is observed at weeks $8-11$, followed by the organization of muscle layers and ICC network in human embryos [25]. The expressions of c-Kit, platelet-derived growth factor, and activin are associated with the differentiation of ICCs or smooth muscle cells [26-29]. From these reports, a mechanism is 
suggested to the effect that activin enhances ICC/smooth muscle cell precursor cells. After that, a distinctive differentiation of ICC from smooth muscle cells is provided by c-Kit signals; this differentiaton is also enhanced by activin [21]. In future studies, a mechanism of activin activation in ES gut should be required for the effective formation of ES gut.

\section{Acknowledgment}

This study was supported in part by a Grant-in-Aid for Scientific Research from the Japan Society for the Promotion of Science.

\section{References}

1 Keller GM: In vitro differentiation of embry onic stem cells. Curr Opin Cell Biol 1995;7: 862-869.

2 Takaki M, Nakayama S, Misawa H, Nakagawa T, Kuniyasu H: In vitro formation of enteric neural network structure in a gut-like organ differentiated from mouse embryonic stem cells. Stem Cells 2006;24:1414-1422.

- 3 Ishikawa T, Nakayama S, Nakagawa T, Horiguchi K, Misawa H, Kadowaki M, Nakao A, Inoue S, Komuro T, Takaki M: Characterization of in vitro gutlike organ formed from mouse embryonic stem cells. Am J Physiol Cell Physiol 2004;286:C1344-C1352.

4 Rodaway A, Patient R: Mesendoderm: an ancient germ layer? Cell 2001;105:169-172.

5 Wells JM, Melton DA: Vertebrate endoderm development. Annu Rev Cell Dev Biol 1999; 15:393-410

6 Lees C, Howie S, Sartor RB, Satsangi J: The hedgehog signalling pathway in the gastrointestinal tract: implications for development, homeostasis, and disease. Gastroenterology 2005;129:1696-1710.

7 Niwa H, Miyazaki J, Smith AG: Quantitative expression of OCT-3/4 defines differentiation, dedifferentiation or self-renewal of ES cells. Nat Genet 2000;24:372-376.

8 Robertson EJ: Embryo-Derived Stem Cell Lines. Washington, IRL, 1987.

-9 Hooper M, Hardy K, Handyside A, Hunter S, Monk M: HPRT-deficient (Lesch-Nyhan) mouse embryos derived from germline colonization by cultured cells. Nature 1987;326: 292-295.

10 Yamada T, Yoshikawa M, Takaki M, Torihashi S, Kato Y, Nakajima Y, Ishizaka S, Tsunoda Y: In vitro function gut-like organ formation from mouse embryonic stem cells. Stem Cells 2002;20:41-49.

-11 van Eyll JM, Pierreux CE, Lemaigre FP, Rousseau GG: SHH-dependent differentiation of intestinal tissue from embryonic pancreas by activin A. J Cell Sci 2004;117:20772086.
12 Mfopou JK, De Groote V, Xu X, Heimberg H, Bouwens L: Sonic hedgehog and other soluble factors from differentiating embryoid bodies inhibit pancreas development. Stem Cells 2007;25:1156-1165.

13 Hinnebusch BF, Siddique A, Henderson JW, Malo MS, Zhang W, Athaide CP, Abedrapo MA, Chen X, Yang VW, Hodin RA: Enterocyte differentiation marker intestinal alkaline phosphatase is a target gene of the gutenriched Kruppel-like factor. Am J Physiol Gastrointest Liver Physiol 2004;286:G23G30.

14 Ueda T, Yamada T, Hokuto D, Koyama F, Kasuda S, Kanehiro H, Nakajima Y: Generation of functional gut-like organ from mouse induced pluripotent stem cells. Biochem Biophys Res Commun 2010;391:38-42.

15 Ishizuya-Oka A, Hasebe T: Sonic hedgehog and bone morphogenetic protein- 4 signaling pathway involved in epithelial cell renewal along the radial axis of the intestine. Digestion 2008;77:42-47.

16 van den Brink GR: Hedgehog signaling in development and homeostasis of the gastrointestinal tract. Physiol Rev 2007;87:13431375.

$\checkmark 17$ Henry GL, Brivanlou IH, Kessler DS, Hemmati-Brivanlou A, Melton DA: TGF- $\beta$ signals and a pattern in Xenopus laevis endodermal development. Development 1996; 122:1007-1015.

18 Geske MJ, Zhang X, Patel KK, Ornitz DM, Stappenbeck TS: Fgf9 signaling regulates small intestinal elongation and mesenchymal development. Development 2008;135: 2959-2968.

$\checkmark 19$ Smith JC, Price BM, Van Nimmen K, Huylebroeck D: Identification of a potent Xenopus mesoderm-inducing factor as a homologue of activin A. Nature 1990;345:729731.

20 Kubo A, Shinozaki K, Shannon JM, Kouskoff V, Kennedy M, Woo S, Fehling HJ, Keller G: Development of definitive endoderm from embryonic stem cells in culture. Development 2004;131:1651-1662.
21 Coutts SM, Childs AJ, Fulton N, Collins C, Bayne RA, McNeilly AS, Anderson RA: Activin signals via SMAD2/3 between germ and somatic cells in the human fetal ovary and regulates kit ligand expression. Dev Biol 2008;314:189-199.

22 Takaki M: Gut pacemaker cells: the interstitial cells of Cajal (ICC). J Smooth Muscle Res 2003;39:137-161.

23 Takaki M, Misawa H, Shimizu J, Kuniyasu $\mathrm{H}$, Horiguchi K: Inhibition of gut pacemaker cell formation from mouse ES cells by the c-kit inhibitor. Biochem Biophys Res Commun 2007;359:354-359.

24 Young HM: Embryological origin of interstitial cells of Cajal. Microsc Res Tech 1999;47: 303-308.

-25 Wallace KN, Akhter S, Smith EM, Lorent K, Pack M: Intestinal growth and differentiation in zebrafish. Mech Dev 2005;122:157173.

26 Beckett EA, Ro S, Bayguinov Y, Sanders KM, Ward SM: Kit signaling is essential for development and maintenance of interstitial cells of Cajal and electrical rhythmicity in the embryonic gastrointestinal tract. Dev Dyn 2007;236:60-72.

-27 Kurahashi M, Niwa Y, Cheng J, Ohsaki Y, Fujita A, Goto H, Fujimoto T, Torihashi S: Platelet-derived growth factor signals play critical roles in differentiation of longitudinal smooth muscle cells in mouse embryonic gut. Neurogastroenterol Motil 2008;20:521531.

28 Topouzis S, Majesky MW: Smooth muscle lineage diversity in the chick embryo: two types of aortic smooth muscle cell differ in growth and receptor-mediated transcriptional responses to transforming growth factor- $\beta$. Dev Biol 1996;178:430-445.

-29 Kanzaki M, Nobusawa R, Mogami H, Yasuda H, Kawamura N, Kojima I: Production of activin A and follistatin in cultured rat vascular smooth muscle cells. Mol Cell Endocrinol 1995;108:11-16. 